\title{
Improved Lower Bounds for the Link Length of Rectilinear Spanning Paths in Grids ${ }^{1}$
}

\author{
Michael J. Collins and Bernard M.E. Moret \\ Department of Computer Science \\ University of New Mexico \\ Albuquerque, NM 87131 \\ mcollins@cs.unm.edu and moret@cs.unm.edu
}

\begin{abstract}
We give improved lower bounds on the link length of spanning paths in a $d$-dimensional grid of size $n$. Kranakis et al. conjectured that the optimal link length is $\left(1+\frac{1}{d-1}\right) n^{d-1}$, but gave lower bounds not substantially larger than $n^{d-1}$; we give a lower bound of $\left(1+\frac{1}{2 d}\right) n^{d-1}$ for all $d>2$.
\end{abstract}

\section{Introduction}

Let $G_{n}^{d}$ be the $d$-dimensional grid of size $n$, i.e., the set of points in $R^{d}$ with integer coordinates in the range 1 to $n$ inclusive. We consider paths that contain all points of the grid and consist entirely of line segments parallel to one of the coordinate axes. These segments are called links; the length of a link is defined to be the number of grid points contained in the link. The link length of a path $P$, denoted $s(P)$, is the number of links that make up $P$, or one plus the number of turns in the path. The link length of the $d$-dimensional grid of size $n$, denoted $s\left(G_{n}^{d}\right)$, is the minimum $s(P)$ over all such paths $P$. Kranakis et al. [1] gave lower bounds on the link length of these grids and also gave a procedure to construct spanning paths with nearly minimal (or perhaps truly minimal) number of links. We give substantial improvements on their lower bounds.

We proceed in two steps: first we prove a crucial lemma that relates the link length of a grid to the maximal length of a fraction of the links; then we use this lemma to derive our bounds by proving a second crucial lemma about the relationship between successively larger subgrids of the given grid.

\section{The Three-Dimensional Case}

We begin with the three-dimensional case. Trivially we have $s\left(G_{n}^{3}\right) \geq n^{2}$, since there are $n^{3}$ points and each segment can cover at most $n$ points. Kranakis et al. derived the lower bound

$$
(1.0232) n^{2} \leq s\left(G_{n}^{3}\right)
$$

\footnotetext{
${ }^{1}$ Work supported by the Office of Naval Research under grants N00014-97-1-0244 and N00014-95$1-1010$
} 
and gave an algorithm to construct a spanning path with a link length of $\frac{3}{2} n^{2}+n-1$, so that we can write

$$
(1.0232) n^{2} \leq s\left(G_{n}^{3}\right) \leq \frac{3}{2} n^{2}+n-1
$$

Thus the problem is to improve the lower bound by raising the constant $c$ that can be placed in front of the $n^{2}$. We obtain below a value of $\frac{7}{6}$.

We begin with the crucial lemma on the relationship between link lengths and number of links.

Lemma 1. Suppose that a spanning path $P$ of $G_{n}^{3}$ has $r$ pairwise-disjoint sets of links $S_{1}, \ldots, S_{r}$ such that, for each $i, S_{i}$ contains at least $\alpha_{i} n^{2}$ links and each such link has length at most $\beta_{i} n$. Then we have

$$
s(P) \geq\left(1+\sum_{i=1}^{r} \alpha_{i}\left(1-\beta_{i}\right)\right) n^{2}
$$

Proof: For each $i$, the links in set $S_{i}$ can cover at most $\alpha_{i} \beta_{i} n^{3}$ points, so the $\sum \alpha_{i} n^{2}$ links in these sets cover a total of $\sum \alpha_{i} \beta_{i} n^{3}$ points. The remaining $\left(1-\sum \alpha_{i} \beta_{i}\right) n^{3}$ points need at least $\left(1-\sum \alpha_{i} \beta_{i}\right) n^{2}$ links, for a total of $\left(1-\sum \alpha_{i} \beta_{i}+\sum \alpha_{i}\right) n^{2}=$ $\left(1+\sum \alpha_{i}\left(1-\beta_{i}\right)\right) n^{2}$ links.

Q.E.D.

Suppose that $n$ is odd-the proof for even values of $n$ is similar. Consider a sequence of $\frac{n-1}{2}$ cubes with sides $3,5,7, \ldots, n$ nested one inside the next, centered within $G_{n}^{3}$. Let $Q_{i}$ be the set of points in the $i$ th cube; for instance, $Q_{1}$ is the set of points of the $3 \times 3 \times 3$ cube centered within $G_{n}^{3}$. Note that, on any spanning path of $G_{n}^{3}$, any link that intersects $Q_{i}$ (i.e., that covers at least one point of $Q_{i}$ ) must be followed immediately by a link of length at most $\frac{n+1}{2}+i$; this second link is aligned with a different axis of the grid and its length cannot exceed the side of the subcube plus the distance from the subcube to the edge of the grid.

Our next crucial lemma establishes a relationship between successively nested subgrids and disjoint subsets of links.

Lemma 2. In any spanning path there must exist disjoint sets of links $S_{i}$, for $1 \leq$ $i \leq \frac{n-1}{2}$, such that $S_{i}$ contains at least $8 i$ links, each of which intersects $Q_{i}$.

Proof: We prove this claim by induction. For $i=1$, the claim is trivially true: a minimum of 9 links is required to cover the $3 \times 3 \times 3$ cube. Suppose then that we have such sets for $i=1, \ldots, k-1$; these sets contain $\sum_{i=0}^{k-1} 8 i=4 k^{2}-4 k$ links, which together cover at most $\left(4 k^{2}-4 k\right)(2 k+1)$ points of $Q_{k}$. But $Q_{k}$ contains $(2 k+1)^{3}$ points, so that $Q_{k}$ still has

$$
(2 k+1)^{3}-\left(4 k^{2}-4 k\right)(2 k+1)=(2 k+1)(8 k+1)
$$


points not covered by any of the links in $\cup_{1}^{k-1} S_{i}$. Since no single link can cover more than $2 k+1$ points of $Q_{k}$, there must be at least $8 k$ other links that intersect $Q_{k}$; these $8 k$ links can be taken to form $S_{k}$.

Q.E.D.

Now each link in $S_{i}$ must be followed by an orthogonal link of length not exceeding $\frac{n+1}{2}+i$; moreover, if two sets of links are disjoint, then the sets of their successors must also be disjoint. Thus we can apply Lemma 1 with $\alpha_{i}=\frac{8 i}{n^{2}}$ and $\beta_{i}=\frac{1}{2}+\frac{i}{n}+\frac{1}{2 n}$, yielding the constant

$$
c \geq 1+\sum_{i=1}^{(n-1) / 2} \frac{8 i}{n^{2}}\left(\frac{1}{2}-\frac{i}{n}-\frac{1}{2 n}\right)=\frac{7}{6}-O\left(\frac{1}{n}\right)
$$

We have proved the following theorem.

Theorem 1. $\forall n, s\left(G_{n}^{3}\right) \geq \frac{7}{6} n^{2}$

\section{The $d$-Dimensional Case}

Our approach easily generalizes to $d$ dimensions. As before we can decompose $G_{n}^{d}$ (where $n$ is again assumed to be odd) into a nested sequence of hypercubes of sides $3,5,7, \ldots, n$. Again let $Q_{i}$ be the set of points in the $i$ th hypercube. As before, a link intersecting $Q_{i}$ must be immediately followed by a link of length not exceeding $\frac{n}{2}+i$.

We begin by generalizing Lemma 2 .

Lemma 3. In any spanning path there must exist $\frac{n-1}{2}$ disjoint sets of links $S_{1}, \ldots, S_{\frac{n-1}{2}}$ such that $S_{i}$ contains $(2 i+1)^{d-1}-(2 i-1)^{d-1}$ links, each of which intersects $Q_{i}$.

Proof: The claim is trivial for $i=1$ : the hypercube of $3^{d}$ points needs a minimum of $3^{d-1}$ links to cover all of its points. Suppose then that we have such sets for $i=1, \ldots, k-1$. These sets contain

$$
\sum_{i=1}^{k-1}\left((2 i+1)^{d-1}-(2 i-1)^{d-1}\right)=(2 k-1)^{d-1}-1
$$

links, which together cover at most $(2 k+1)\left((2 k-1)^{d-1}-1\right)$ points of $Q_{k}$. But $Q_{k}$ has $(2 k+1)^{d}$ points, so that the links in $\cup_{1}^{k-1} S_{i}$ leave uncovered at least

$$
(2 k+1)^{d}-\left((2 k+1)(2 k-1)^{d-1}-1\right)=(2 k+1)\left((2 k+1)^{d-1}-(2 k-1)^{d-1}+1\right)
$$

points of $Q_{k}$. Since no link may cover more than $(2 k+1)$ points of $Q_{k}$, it follows that there must be at least $(2 k+1)^{d-1}-(2 k-1)^{d-1}$ other links that intersect $Q_{k}$; these links can be taken to form $S_{k}$.

Q.E.D. 
As before we may apply Lemma 1 (which readily generalizes to $d$ dimensions) with $\alpha_{i}=\frac{\left|S_{i}\right|}{n^{d-1}}$ and $\beta_{i}=\frac{1}{2}+\frac{i}{n}+\frac{1}{2 n}$. Disregarding terms of lower order, we have $\left|S_{i}\right|=$ $(d-1) 2^{d-1} i^{d-2}$ and thus can write (for $n$ going to infinity)

$$
c \geq 1+\sum_{i=1}^{n / 2} \frac{(d-1) 2^{d-1} i^{d-2}}{n^{d-1}}\left(\frac{1}{2}-\frac{i}{n}-\frac{1}{2 n}\right) \asymp 1+\frac{1}{2 d}
$$

We have proved the generalization of Theorem 1.

Theorem 2. $\forall n, \forall d>2, s\left(G_{n}^{d}\right) \geq\left(1+\frac{1}{2 d}\right) n^{d-1}-O\left(n^{d-2}\right)$

Kranakis et al. had given the asymptotic lower bound

$$
\frac{s\left(G_{n}^{d}\right)}{n^{d-1}} \geq 1+\frac{1}{2}\left(1-\exp \left(\frac{-1}{d(d-1)}\right)\right)
$$

and given an algorithm that, asymptotically for any $\epsilon<1$, produces a spanning path obeying

$$
\frac{s\left(G_{n}^{d}\right)}{n^{d-1}} \leq 1+\frac{1}{2(d-3)^{1-\epsilon}}+\exp \left(-(d-3)^{\epsilon}\right)
$$

which implies that there exists some positive constant $k$ with

$$
\frac{s\left(G_{n}^{d}\right)}{n^{d-1}} \leq 1+\frac{k+\log d}{2(d-3)}
$$

They also conjectured that the asymptotically optimal value is $1+\frac{1}{d-1}$. Our lower bound takes us halfway to this conjectured value.

\section{Conclusion}

We have given much improved lower bounds on the link length of spanning paths for $d$-dimensional grids. None of our results require that the spanning path avoid self-intersections, nor do we require that the path stay inside the grid. In the case of $G_{n}^{d}$, it is not clear that these assumptions would matter: the paths constructed by the procedure given by Kranakis et al. are the shortest known and do not intersect themselves nor venture outside the grid. The same assumptions clearly do matter if we seek spanning paths for arbitrary sets of points; our current work is aimed at developing methods to compute lower bounds on the link length of spanning paths for rectilinear polyhedral grids.

\section{References}

[1] Kranakis, E., D. Krizanc, and L. Meertens, "Link length of rectilinear Hamiltonian tours on grids," Ars Combinatoria 38 (1994), p. 177. 